\title{
Quantum Chemical Topology as a Theory of Open Quantum Systems
}

\author{
A. Martín Pendás* and E. Francisco* \\ Departamento de Química Física y Analítica. Facultad de Química. Universidad de \\ Oviedo. 33006 Oviedo. Spain. \\ E-mail: ampendas@.uniovi.es
}

\begin{abstract}
Although real space regions have been widely used in theoretical chemistry, not much effort has been devoted to treat them as open quantum systems. We embrace this task here, finding closed expressions for the density operator of a quantum subsystem in real space by tracing out the degrees of freedom in its complementary region. Our results are then linked to previous knowledge. For single-determinant descriptions it is shown that the entanglement orbitals coincide with Ponec's domain natural orbitals. In general, the subsystem density operator is written as a direct sum of fixed number of electron sectors, with weights that turn out to be equal to those found within the theory of electron distribution functions. As a computational application we show how to obtain the global first order density matrix of a subsystem and its eigensolution in a couple of toy systems. In the multi-determinant wave function case, the domain natural orbitals defined through this open systems approach do not coincide with those of Ponec, and, contrary to the latter, have always strictly positive occupations.
\end{abstract}




\section{Introduction}

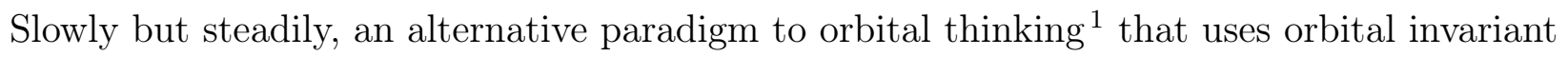
quantities is gaining popularity in the theory of the chemical bond. Quantum chemical topology (QCT) ${ }^{2}$ as this set of techniques are collectively known, uses the 3D physical space (or real space) as its favourite playground. This is particularly rewarding for the practicing chemist who, despite of the orbital varnish that permeates chemical modeling, still thinks and designs in real space, e.g. via arrow pushing. ${ }^{3}$ QCT spatial descriptors are built from reduced (spatial) density matrices (RDMs) and do not depend on the underlying methodology used to obtain the wavefunction of the system. In many cases, the space is divided into regions endowed with chemical meaning, like atoms, cores, bonds, lone-pairs, etc. through the topology induced by a relevant scalar or vector field. In the quantum theory of atoms in molecules (QTAIM) proposed by Bader and coworkers, ${ }^{4}$ for instance, it is the electron density $\rho$ which is used to provide quantum atoms which, being subsystems of the full quantum system, are open quantum objects. This fact has been acknowledged in the QTAIM since its inception.

Density matrices traced out in real space have become, on the other hand, a basic tool in condensed matter physics, ${ }^{5}$ and concepts such as the entanglement spectrum of a subsystem ${ }^{6}$ or its entanglement entropy ${ }^{7}$ have been proposed to characterize many of their features, from the coupling of two systems to the systematization of the nature of topological insulators. ${ }^{8}$ In its turn, the use of subsystems in theoretical chemistry, although relatively recent, has also found its way. Methods such as the density matrix embedding theory (DMET) ${ }^{9,10}$ fully rest on subsystem traces, and relatively older procedures, like the density matrix renormalization group techniques (DMRG), ${ }^{11}$ also make use of the Schmidt decomposition that is basic to the treatment of open systems. Consideration of entanglement measures to understand correlation effects ${ }^{12}$ or more general chemical phenomena have also appeared. ${ }^{13}$ However, direct application of these ideas to chemical bonding is scarce in the physical community, although Tubman and Yang (TY in the following) have acknowledged the clear relation 
between open quantum systems (OQSs) and chemical bonding in the last years. ${ }^{14,15}$

Here we show how the original insight of Bader regarding the open nature of atoms-inmolecules may be fully formalized in terms of concepts borrowed from the theory of OQSs. In this journey, we will prove that the statistics of the electron population, as determined by the electron distribution functions (EDFs) already introduced in $\mathrm{QCT},{ }^{16}$ is immediately recovered from the entanglement spectrum of the subsystem. We will also show that for single determinant descriptions, and for a partition of the space into two regions, Ponec's domain natural orbitals (DNOs), ${ }^{17,18}$ which have been known for years, come out naturally from the Schmidt's decomposition of a Slater determinant. This link, which is in fact equivalent to the analysis provided by TY, has passed unnoticed (as far as we know) in the chemical literature. As we will highlight, many of the electron delocalization (thus bond-order) measures used in chemistry are also entanglement measurements. We also generalize the TY finding that chemical bonding reflects the quantum mechanical entanglement of two spatial regions. Our insights show that most of the modern statistical (through EDFs) and energetic (through the interacting quantum atoms approach, IQA ${ }^{19,20}$ ) descriptors used in the QTAIM admit an OQS interpretation.

\section{Composite quantum systems}

We succinctly review here a few informal concepts in the theory of open quantum systems to introduce our nomenclature. Longer accounts may be found in the texts of Breuer and Petruccione ${ }^{21}$ or of Nielsen and Chuang. ${ }^{22}$

Let the state vector of an $N$-electron pure state be $|\Psi(1, \ldots, N)\rangle$. The density operator defined as $\hat{\rho}=|\Psi\rangle\langle\Psi|$ enjoys the following properties: (i) $\operatorname{Tr} \hat{\rho}=1$; (ii) $\hat{\rho}^{2}=\hat{\rho}$; (iii) $\operatorname{Tr}(\hat{A} \hat{\rho})=$ $\langle\hat{A}\rangle$. For a mixed state constructed as an ensemble of systems found in state $\left|\Psi_{i}\right\rangle$ with

probability $p_{i}, \hat{\rho}=\sum_{i}\left|\Psi_{i}\right\rangle p_{i}\left\langle\Psi_{i}\right|$, and: (i) $\operatorname{Tr} \hat{\rho}=1$; (ii) $\operatorname{Tr}\left(\hat{\rho}^{2}\right) \leq 1$, the equality being achieved if, and only if, the state is pure. In the case of a mixed state, several entropy 
measures, like the von Neumann entropy $S=-\operatorname{Tr} \rho \ln \rho=-\sum_{i} p_{i} \ln p_{i}$ or the linear entropy, $S_{l}=\operatorname{Tr}\left(\rho-\rho^{2}\right), 0 \leq S_{l} \leq 1$, are often used

Now consider two systems $S^{1}, S^{2}$ with Hilbert spaces $H^{1}, H^{2}$, respectively. The composite system $S$ lives in the tensor product spate $H^{1} \otimes H^{2}$. If $\left\{\left|\phi_{i}^{1}\right\rangle\right\}$ and $\left\{\left|\phi_{j}^{2}\right\rangle\right\}$ are orthonormal bases in $H^{1}, H^{2}$ (countable bases have been assumed, but the results are general), then a state in $S$ can be written as $|\Psi\rangle=\sum_{i j} a_{i j}\left|\phi_{i}^{1}\right\rangle \otimes\left|\phi_{j}^{2}\right\rangle$. The subsystems are uncorrelated if $\hat{\rho}=\hat{\rho}^{1} \otimes \hat{\rho}^{2}$. Otherwise they are said to be entangled. If we are interested in observables of subsystem $S^{1}$ (through operators $A^{1}$ ), we first extend these operators to the full system. For instance, if $A^{1}$ is an operator in $S^{1}$, its extension in $S$ is defined as $A=A^{1} \otimes 1^{2}$. Then we notice that the expectation value of this operator can be obtained from a reduced density operator in subsystem $S^{1}$ defined by taking the partial trace of $\hat{\rho}$ over subsystem $S^{2}$. $\langle A\rangle=\operatorname{Tr}(A \hat{\rho})=\operatorname{Tr} A^{1} \hat{\rho}^{1}$, where $\hat{\rho}^{1}=\operatorname{Tr}_{2} \hat{\rho}$. If a system is in a pure state, any subsystem is not, in general. For a pure state, the entropy of a subsystem's density operator is called its entaglement entropy, and its spectrum the entanglement spectrum. The subsystem (or reduced) density operator will play a fundamental role in the following.

If, as before, we take $|\Psi\rangle=\sum_{i j} a_{i j}\left|\phi_{i}^{1}\right\rangle \otimes\left|\phi_{j}^{2}\right\rangle$ then, independently of the dimensions of $S^{1}, S^{2}$, the matrix a can be subjected to a singular value decomposition, so that $a=u \alpha v$, where $\alpha$ is a diagonal matrix and $u=u^{+}, v=v^{+}$are unitary matrices. With this, $|\Psi\rangle=$ $\sum_{i j k} u_{i j} \alpha_{j} v_{j k}\left|\phi_{i}^{1}\right\rangle \otimes\left|\phi_{k}^{2}\right\rangle$, and we may define new orthonormal bases $\left|\chi_{j}^{1}\right\rangle=\sum_{i} u_{i j}\left|\phi_{i}^{1}\right\rangle,\left|\chi_{j}^{2}\right\rangle=$ $\sum_{k} v_{j k}\left|\phi_{k}^{2}\right\rangle$ so that $|\Psi\rangle=\sum_{i} \alpha_{i}\left|\chi_{i}^{1}\right\rangle \otimes\left|\chi_{i}^{2}\right\rangle$. This is the famous Schmidt decomposition. ${ }^{23}$ The number of non-zero $\alpha_{i}$ values is called the Schmidt number, and it may be shown that $\sum_{i} \alpha_{i}^{2}=1$

If $S$ is in a pure state, then $\hat{\rho}^{1}=\sum_{i} \alpha_{i}^{2}\left|\chi_{i}^{1}\right\rangle\left\langle\chi_{i}^{1}\right|$, and $\hat{\rho}^{2}=\sum_{i} \alpha_{i}^{2}\left|\chi_{i}^{2}\right\rangle\left\langle\chi_{i}^{2}\right|$, so that the spectrum of both subystems is identical. As we will show, these states are exactly correlated with the well known Ponec's DNOs of computational chemistry. ? ? It can be shown that $S$ is a product state if $S^{1}, S^{2}$ have Schmidt number equal to one, and that $S(\rho) \leq S\left(\rho^{1}\right)+S\left(\rho^{2}\right)$. Moreover, as it can immediately be found from the Schmidt decomposition, if a pure system 
is divided into two subsystems, the entropy of each of them is equal (and zero if the state is a product state).

\section{QCT domains as open systems}

As briefly commented, it is very often the case in QCT that the physical space $R^{3}$ is exhaustively partitioned into a set of non-overlapping spatial domains $A_{i}, \bigcup_{i} A_{i}=R^{3}$. For instance, in the QTAIM these regions are associated to the attraction basins of the maxima of the electron density $\rho$, which usually coincide with the nuclear cusps. ${ }^{4}$ It is easy to show that these QTAIM domains, or QTAIM atoms, are separated by local zero-flux surfaces of the $\boldsymbol{\nabla} \rho$ gradient field, such that $\boldsymbol{\nabla} \rho(\boldsymbol{r}) \cdot \boldsymbol{n}=0$ at each point $\boldsymbol{r}$ of the interatomic surface characterized by normal vector $\boldsymbol{n}$.

From the spatial standpoint of QCT, the open nature of any of these subsystems is clear. Since the number operator does only commute with the Hamiltonian of the full system, the number of electrons of a subsystem ceases to be a good quantum number, thus its reduced density must necessarily correspond to a mixed state.

The full density operator $\hat{\rho}$ for an $N$-electron molecular system in a pure state can be written as $\hat{\rho}=\Psi^{\star}\left(\boldsymbol{x}_{1}^{\prime} \ldots, \boldsymbol{x}_{N}^{\prime}\right) \Psi\left(\boldsymbol{x}_{1} \ldots, \boldsymbol{x}_{N}\right)$. We use $\boldsymbol{x}=\boldsymbol{r} \sigma$ to denote a general spin $(\sigma)$ $\operatorname{spatial}(\boldsymbol{r})$ coordinate. Let us now consider a real space subregion $A$, so that the space $R^{3}$ is divided in two domains $A$ and $\bar{A}=B, A \cup B=R^{3}$. We introduce the indicator function of a domain (a Heaviside-like domain weight function) $\omega_{\Omega}$ such that $\omega_{\Omega}(\boldsymbol{x})=0$ and $\omega_{\Omega}(\boldsymbol{x})=1$ for $\boldsymbol{x} \notin \Omega$ and $\boldsymbol{x} \in \Omega$, respectively (with an equivalent definition for the primed variables). It is customary to define also $\omega_{\Omega}(\boldsymbol{x})=1 / 2$ for points exactly at the boundary that separates the $\mathrm{A}$ and $\mathrm{B}$ domains, although this is unimportant here. Indicator functions have been extensively used in QCT before. ${ }^{19}$

We can now define an $N$-electron spatial projection using $1^{N}=\prod_{i=1}^{N}\left[\omega_{A}\left(\boldsymbol{x}_{i}\right)+\omega_{B}\left(\boldsymbol{x}_{i}\right)\right]$. Applying this projection to the $\boldsymbol{x}$ and $\boldsymbol{x}^{\prime}$ coordinates in the the $\hat{\rho}$ operator above, the full 
density operator becomes a sum of $2^{2 N}$ terms in which the primed and unprimed electrons are separated into the $A$ and $B$ spatial domains. The reduced density operator of domain $A$, $\hat{\rho}^{A}$, is obtained from $\hat{\rho}$ by performing a spatial partial trace over the $B$ region, with the usual $\boldsymbol{x}_{i}^{\prime} \rightarrow \boldsymbol{x}_{i}$ identification before integration. When this is done, only those terms in which the domain assignment of each primed and unprimed pairs $\boldsymbol{x}_{i}^{\prime}, \boldsymbol{x}_{i}$ coincide survive, leaving only $2^{N}$ terms. Each corresponds to a given number of alpha and beta electrons in domain $A$, a spin sector in a common notation in the theory of OQSs. In order to simplify, we will group spin sectors in spinless sectors. All our arguments are immediately generalized to the spin resolved case. Using electron indistinguishability, the $2^{N}$ terms can be classified into $N+1$ sectors, using a common notation in the theory of OQSs, each contaning a different number of electrons in $A$, irrespectively of their spin:

$$
\hat{\rho}^{A}=\bigoplus_{n=0}^{N} \hat{\rho}_{n}^{A},
$$

where $\hat{\rho}_{0}^{A}=\int_{B} \Psi^{\star}\left(\boldsymbol{x}_{1} \ldots \boldsymbol{x}_{N}\right) \Psi\left(\boldsymbol{x}_{1} \ldots \boldsymbol{x}_{N}\right) d \boldsymbol{x}_{1} \ldots d \boldsymbol{x}_{N}$ and, for $n \geq 1$

$$
\begin{array}{r}
\hat{\rho}_{n}^{A}\left(\boldsymbol{x}_{i \leq n} ; \boldsymbol{x}_{i \leq n}^{\prime}\right)=\prod_{i=1}^{n} \omega_{A}\left(\boldsymbol{x}_{i}^{\prime}\right) \omega_{A}\left(\boldsymbol{x}_{i}\right) \times \\
\times\left(\begin{array}{c}
N \\
n
\end{array}\right) \int_{B} \Psi^{\star}\left(\boldsymbol{x}_{i \leq n}^{\prime}, \boldsymbol{x}_{i>n}\right) \Psi\left(\boldsymbol{x}_{i \leq n}, \boldsymbol{x}_{i>n}\right) d \boldsymbol{x}_{i>n},
\end{array}
$$

with $\boldsymbol{x}_{i \leq n}=\boldsymbol{x}_{1} \ldots \boldsymbol{x}_{n}$ and $\boldsymbol{x}_{i>n}=\boldsymbol{x}_{n+1} \ldots \boldsymbol{x}_{N}$, for instance. Subsystem $A$ is thus described by a mixed density operator with $N+1$ possible values for its number of electrons. Each sector is described by its own density operator $\hat{\rho}_{n}^{A}$ which can be diagonalized to obtain its spectrum, composed of eigenvalues $0 \leq n_{i} \leq 1$. As we will explain, these eigenvalues are intimately linked to traditional bonding concepts. Each $\hat{\rho}_{n}^{A}$ can also be interpreted in real space as a (not-normalized) $n$-electron density matrix in its sector, $\rho_{n}^{A}$.

Since we are dealing with pure states and the squares of the Schmidt numbers add to one, $\operatorname{Tr} \hat{\rho}^{A}=1$, this meaning that the individual sector $\hat{\rho}_{n}^{A}$ 's are not normalized. We can 
check that

$$
\operatorname{Tr} \hat{\rho}_{n}^{A}=\left.\int_{A} \rho_{n}^{A}\left(\boldsymbol{x}_{i \leq n} ; \boldsymbol{x}_{i \leq n}^{\prime}\right)\right|_{\boldsymbol{x}_{i}^{\prime} \rightarrow \boldsymbol{x}_{i}} d \boldsymbol{x}_{i \leq n}=\int_{A} \rho_{n}^{A}\left(\boldsymbol{x}_{i \leq n}\right) d \boldsymbol{x}_{i \leq n}=p^{A}(n),
$$

where $p^{A}(n)$ is the probability that $n$ and only $n$ electrons reside in spatial domain $A$. This result, now well known, goes back to Daudel's theory of loges, ${ }^{24}$ and has been used to define maximum probability domains, ${ }^{25}$ a promising tool in chemical bonding, and to construct a statistical image of bonding through the use of electron distribution functions (EDFs). ${ }^{16,26}$ Actually, when a system is divided into two $A, B$ regions, $p^{A}(n)$ is equivalent to the full EDF, $p^{A}(n)=p\left(n_{A}=n, n_{B}=N-n\right)$. It has been shown that $\mathrm{A}-\mathrm{B}$ covalency can be interpreted in terms of the fluctuation of their electron population and that, for instance, a measure of the $\mathrm{A}-\mathrm{B}$ covalent bond order is provided by the so-called delocalization index, $\delta(A, B)=-2 \operatorname{cov}\left(n_{A}, n_{B}\right)$. In the present context, this information is thus encoded in the entanglement spectrum, and each sector corresponds to a real space resonance structure, as already defined. ${ }^{27}$

It is practical to use in the following normalized sector densities, $\tilde{\rho}_{n}^{A}=\left[p^{A}(n)\right]^{-1} \rho_{n}^{A}$, that satisfy $\operatorname{Tr} \tilde{\rho}_{n}^{A}=1$ so that

$$
\hat{\rho}^{A}=\bigoplus_{n=0}^{N} p^{A}(n) \tilde{\rho}_{n}^{A}
$$

Doing so we can deal with each $\tilde{\rho}_{n}^{A}$ as a pseudo pure system operator, which we can manipulate on its own in each sector. Except when confusion may arise, we will drop, from now on, the tilde on the normalized operators.

\section{Sector reduced density matrices}

As already commented, each of the (now normalized) $\hat{\rho}_{n}^{A}(n \geq 1)$ operators can be interpreted in real space as the $n$-th order spatial density matrix in its $n$-electron sector. Some of these electrons may be integrated out, defining in this way sector reduced density matrices (RDMs). 
Using standard notation, the $m$-th order RDM in sector $n$ is introduced as,

$$
\rho_{n}^{A, m}\left(\boldsymbol{x}_{i \leq m} ; \boldsymbol{x}_{i \leq m}^{\prime}\right)=\left.\frac{n !}{(n-m) !} \int \rho_{n}^{A}\left(\boldsymbol{x}_{i \leq n} ; \boldsymbol{x}_{i \leq n}^{\prime}\right)\right|_{\boldsymbol{x}_{i}^{\prime} \rightarrow \boldsymbol{x}_{i}} d \boldsymbol{x}_{i>m}
$$

with trace $\operatorname{Tr} \rho_{n}^{A, m}=n ! /(n-m) !$, and spinless $m$-th order RDM given by $\rho_{n}^{A, m}\left(\boldsymbol{r}_{i \leq m} ; \boldsymbol{r}_{i \leq m}^{\prime}\right)=$ $\left.\int \rho_{n}^{A, m}\left(\boldsymbol{x}_{i \leq m} ; \boldsymbol{x}_{i \leq m}^{\prime}\right)\right|_{\sigma_{i}^{\prime} \rightarrow \sigma_{i}} d \sigma_{i \leq m}$

These RDMs play in each sector the same role as the standard reduced density matrices in the full system. Since we are basically interested in one- and two-electron operators in molecules, the 1- and 2RDMs are particularly important.

Let us take, for instance, a strictly local one-electron operator like the electron density operator, $\hat{n}(\boldsymbol{r})=\sum_{i} \delta\left(\boldsymbol{r}-\boldsymbol{r}_{i}\right)$. We use $\hat{n}$ so that no confusion arises with the system's $\hat{\rho}$. The expectation value of $\hat{n}(\boldsymbol{r})$ is the electron density, $n(\boldsymbol{r})=\rho^{1}(\boldsymbol{r})=\operatorname{Tr}(\hat{n} \hat{\rho})$. Being a one-electron operator, we can use the $1 \mathrm{RDM} \rho^{1}\left(\boldsymbol{r} ; \boldsymbol{r}^{\prime}\right)$ to get its expectation value as $\rho^{1}(\boldsymbol{r})=\operatorname{Tr}\left\{\delta\left(\boldsymbol{r}-\boldsymbol{r}_{1}\right) \rho^{1}\left(\boldsymbol{r}_{1} ; \boldsymbol{r}_{1}^{\prime}\right)\right\}$. Using the sector RDMs, the density at point $\boldsymbol{r}$ in domain $A, \rho^{1}(\boldsymbol{r} \in A)=\rho^{A, 1}(\boldsymbol{r})$ turns out to be

$$
\rho^{A, 1}(\boldsymbol{r})=\sum_{n=1}^{N} p^{A}(n) \operatorname{Tr}\left\{\delta\left(\boldsymbol{r}-\boldsymbol{r}_{1}\right) \rho_{n}^{A, 1}\left(\boldsymbol{r}_{1} ; \boldsymbol{r}_{1}^{\prime}\right)\right\}
$$

which shows that the subsystem's expectation value is a weighted sum of well-defined densities for each sector. This also evidences that the subsystem's projected operator $\hat{n}^{A}$ has a different component in each sector, $\hat{n}^{A}(\boldsymbol{r})=\bigoplus_{n=1}^{N} \hat{n}_{n}^{A}(\boldsymbol{r})$, with $\hat{n}_{n}^{A}(\boldsymbol{r})=\sum_{i=1}^{n} \delta\left(\boldsymbol{r}-\boldsymbol{r}_{i}\right)$. These arguments can be extended easily to local two-electron operators. In other cases, like when dealing with the kinetic energy, the presence of derivatives of the indicator functions has to be dealt with carefully, giving rise to surface terms that are well known to QCT. We will not discuss these subtleties here.

Once the sector RDMs have been appropriately defined, all the standard density matrix machinery is at our disposal. For instance, sector RDMs can be diagonalized to obtain 
sector natural orbitals, geminals, etc. As we will show, these are immediately related to other objects already defined, such as the domain natural orbitals introduced by Robert Ponec in the nineties, ${ }^{28,29}$ or the natural adaptive orbitals, ${ }^{30}$ which promise to obtain new insights in chemical bonding.

Notice finally that Eqs. 5 and 6 admit a simple deconvolution, so that it is easily shown that the weighted sum in Eq. 6 allows us to write $\rho^{A, 1}\left(\boldsymbol{x} ; \boldsymbol{x}^{\prime}\right)=\omega_{A}\left(\boldsymbol{x}^{\prime}\right) \omega_{A}(\boldsymbol{x}) \rho^{1}\left(\boldsymbol{x} ; \boldsymbol{x}^{\prime}\right)$. This result can be generalized to all the other subsystem RDMs.

\section{The single determinant case}

Let us now consider an $N$-electron system in a pure state described by a single-determinant wavefunction (SDW) $|\Psi\rangle,|\Psi\rangle=(N !)^{-1 / 2} \operatorname{det}\left|u_{1}\left(\boldsymbol{x}_{1}\right) \ldots u_{N}\left(\boldsymbol{x}_{N}\right)\right|$. Using the Laplace expansion, $|\Psi\rangle$ can be expanded in terms of its first $n$ rows

$$
|\Psi\rangle=(N !)^{-1 / 2} \sum_{\boldsymbol{k}} \operatorname{det}\left|u_{k_{1}}\left(\boldsymbol{x}_{1}\right) \ldots u_{k_{n}}\left(\boldsymbol{x}_{n}\right)\right| \times \operatorname{det}(n \mid \boldsymbol{k})
$$

where $\boldsymbol{k}$ denotes the ordered set $k_{1}<k_{2}<\cdots<k_{n}$, and $\operatorname{det}(n \mid \boldsymbol{k})$, that depends only on the coordinates $\boldsymbol{x}_{n+1}, \ldots, \boldsymbol{x}_{N}$, is the determinant obtained from $\operatorname{det}\left|u_{1}\left(\boldsymbol{x}_{1}\right) \ldots u_{N}\left(\boldsymbol{x}_{N}\right)\right|$ by deleting the first $n$ rows and the columns $k_{1}, k_{2}, \ldots, k_{n}$. With this $|\Psi\rangle$, the expression for the unnormalized $\hat{\rho}_{n}^{A}$ becomes,

$$
\begin{aligned}
\hat{\rho}_{n}^{A} & =\mathbf{1}_{n}^{\prime A} \mathbf{1}_{n}^{A} \times \sum_{\boldsymbol{k}, \boldsymbol{l}}\left|U_{\boldsymbol{k}}\right\rangle\left\langle U_{\boldsymbol{l}}|\operatorname{det}| S^{B}(\tilde{\boldsymbol{k}} \mid \tilde{\boldsymbol{l}})\right|, \quad \text { where } \\
\left|U_{\boldsymbol{k}}\right\rangle & =(n !)^{-1 / 2}\left|u_{k_{1}}\left(\boldsymbol{x}_{1}\right) \ldots u_{k_{n}}\left(\boldsymbol{x}_{n}\right)\right| \\
\left\langle U_{\boldsymbol{l}}\right| & =(n !)^{-1 / 2}\left|u_{l_{1}}\left(\boldsymbol{x}_{1}^{\prime}\right) \ldots u_{l_{n}}\left(\boldsymbol{x}_{n}^{\prime}\right)\right|
\end{aligned}
$$

we have abbreviated the indicator function $\prod_{i=1}^{n} \omega_{A}\left(\boldsymbol{x}_{i}\right)$ as $\mathbf{1}_{n}^{A}$, and assumed real spinorbitals. $S^{B}$ is the $(N \times N)$ domain overlap matrix (DOM) in $B$, defined as $S_{i j}^{B}=\left\langle u_{i} \mid u_{j}\right\rangle_{B}$, and $S^{B}(\tilde{\boldsymbol{k}} \mid \tilde{\boldsymbol{l}})$ the $(N-n) \times(N-n)$ array obtained from $S^{B}$ by selecting rows $\tilde{\boldsymbol{k}}$ and columns 
$\tilde{\boldsymbol{l}}$, complementary of $\boldsymbol{k}$ and $\boldsymbol{l}$, respectively. Notice that the sector density operator is clearly a mixed state formed from $n$-electron determinants extracted from the original $N$-electron one.

The $n$-electron basis $\left|U_{\boldsymbol{k}}\right\rangle$ is not orthonormal in $A$, since $\boldsymbol{S}_{\boldsymbol{k} \boldsymbol{l}}^{A}=\left\langle U_{\boldsymbol{k}} \mid U_{\boldsymbol{l}}\right\rangle_{A}=\operatorname{det}\left|S^{A}(\boldsymbol{k} \mid \boldsymbol{l})\right|$. It is then convenient to orthonormalize it, for instance via a Löwdin transformation, $\left|\psi_{\boldsymbol{k}}\right\rangle=$ $\left|U_{\boldsymbol{k}}\right\rangle\left(\boldsymbol{S}^{A}\right)^{-1 / 2}$, such that $\left\langle\psi_{\boldsymbol{k}} \mid \psi_{\boldsymbol{l}}\right\rangle_{A}=\delta_{\boldsymbol{k} \boldsymbol{l}}$. The set of $\left|\psi_{\boldsymbol{k}}\right\rangle$ functions constitutes an $n$-electron basis from which the $n$-sector is built. Defining the matrix $\tilde{\boldsymbol{S}}_{\boldsymbol{k l}}^{B}=\operatorname{det}\left|S^{B}(\tilde{\boldsymbol{k}} \mid \tilde{\boldsymbol{l}})\right|$, Eq. 8 becomes

$$
\hat{\rho}_{n}^{A}=\mathbf{1}_{n}^{\prime A} \mathbf{1}_{n}^{A} \times \sum_{k, l}\left|\psi_{\boldsymbol{k}}\right\rangle P_{\boldsymbol{k} l}\left\langle\psi_{\boldsymbol{l}}\right|
$$

where $\boldsymbol{P}_{\boldsymbol{k} \boldsymbol{l}}=\left(\boldsymbol{S}_{\boldsymbol{k} \boldsymbol{l}}^{A}\right)^{1 / 2} \tilde{\boldsymbol{S}}_{\boldsymbol{k} \boldsymbol{l}}^{B}\left(\boldsymbol{S}_{\boldsymbol{k} \boldsymbol{l}}^{A}\right)^{1 / 2}$. The normalized sector density operator can thus be written as

$$
\tilde{\rho}_{n}^{A}=\mathbf{1}_{n}^{\prime A} \mathbf{1}_{n}^{A} \times \sum_{\boldsymbol{k}, \boldsymbol{l}}\left|\psi_{\boldsymbol{k}}\right\rangle \frac{\boldsymbol{P}_{\boldsymbol{k} l}}{\sum_{\boldsymbol{k}} \boldsymbol{P}_{\boldsymbol{k} k}}\left\langle\psi_{\boldsymbol{l}}\right|
$$

with $p^{A}(n)=\sum_{\boldsymbol{k}} \boldsymbol{P}_{\boldsymbol{k} \boldsymbol{k}}$. We can obviously diagonalize $\hat{\rho}_{n}^{A}$ (or $\left.\tilde{\rho}_{n}^{A}\right)$ in the $\left|\psi_{\boldsymbol{k}}\right\rangle$ basis. This is equivalent to diagonalizing the $\boldsymbol{P}$ matrix above. Its eigenvalues are the entanglement spectrum of the sector, $p_{n}^{k}$ :

$$
\hat{\rho}_{n}^{A}=\mathbf{1}_{n}^{\prime A} \mathbf{1}_{n}^{A} \times \sum_{k}\left|\phi_{\boldsymbol{k}}\right\rangle p_{n}^{k}\left\langle\phi_{\boldsymbol{k}}\right|
$$

The $p_{n}^{k}$ are indeed probabilities that add to the overall sector probability $p^{A}(n)$ (or to one if the normalized $\tilde{\rho}_{n}^{A}$ operator is used, with eigenvalues now labelled as $\left.\tilde{p}^{\boldsymbol{k}}(n)\right)$. Each $p_{n}^{\boldsymbol{k}}$ can be interpreted as the probability of finding the subsystem in state $\left|\phi_{\boldsymbol{k}}\right\rangle$. All the sector RDMs can be immediately obtained from the expressions above. For instance, the 1RDM of the $n$-electron sector is obtained from Eq. 12 by tracing out $n-1$ electrons as in Eq. 5, using Slater's rules. 


\subsection{Exploiting the Schmidt decomposition}

When a system is divided into two subsystems $A$ and $B$, important algebraic simplifications based on the Schmidt decomposition appear, since it is possible to find a one-electron basis which is simultaneously orthogonal in $A, B$, and $R^{3}$. This is performed by diagonalizing the $S_{i j}^{A}=\left\langle u_{i} \mid u_{j}\right\rangle_{A}$ matrix by a unitary transformation $\boldsymbol{V}, \boldsymbol{V}^{\dagger} \boldsymbol{S}^{A} \boldsymbol{V}=\operatorname{diag}\left(s_{i}\right)=\boldsymbol{s}$. The new one-electron basis $\left|u^{P}\right\rangle=|u\rangle \boldsymbol{V}$ is obviously orthonormal in $R^{3}$, so that the transformed $|\Psi\rangle$ determinant does not change, and orthogonal both in $A$ and $B,\left\langle u_{i}^{P} \mid u_{j}^{P}\right\rangle_{A}=1-\left\langle u_{i}^{P} \mid u_{j}^{P}\right\rangle_{B}=$ $s_{i} \delta_{i j}$. Eqs. 7 to 13 remain untouched when $|\Psi\rangle$ is rewritten in this $\left|u^{P}\right\rangle$ basis. An orthonormal one-electron basis in $A$ can immediately be constructed from $\left|u^{P}\right\rangle$ as $\left|\tilde{u}^{P}\right\rangle=\left|u^{P}\right\rangle s^{-1 / 2}$. Interestingly, the $s_{i}$ parameters have also a statistical interpretation, being equal to the probability of finding an electron in state $\left|\bar{\psi}_{i}\right\rangle$ in region $A\left(1-s_{i}\right.$ in the case of region $\left.B\right)$. The Löwdin one electron basis used in Eqs. 11 and 12 is a unitary transformation (in $A$ ) of the present basis: $\left|\tilde{u}^{P}\right\rangle=|\psi\rangle \boldsymbol{W}$, with $\boldsymbol{W}=\left(\boldsymbol{S}^{A}\right)^{1 / 2} \boldsymbol{V} \boldsymbol{s}^{-1 / 2}$.

It is particularly important for chemical bonding purposes that the $\left|u^{P}\right\rangle$ basis is exactly that proposed by R. Ponec for single determinants. ${ }^{17,18}$ Ponec's orbitals, called domain natural orbitals (DNOs), have been successfully used to extract chemical information, and have been also interpreted in statistical terms. ${ }^{31}$ It has also been shown that electrons in DNOs behave as effective statistically independent particles in regions $A$ and $B$, and that the electron distribution function can be obtained by direct multiplication of the probabilities (the $s_{i}$ parameters) of finding the electrons in these effective states.

In the DNO basis, the $\tilde{\boldsymbol{S}}_{\boldsymbol{k} \boldsymbol{l}}^{B}$ is diagonal, since if any $l_{i}$ is different from $k_{i}, S^{B}(\tilde{\boldsymbol{k}} \mid \tilde{\boldsymbol{l}})$ will contain one or more zeros in the diagonal and $\operatorname{det}\left|S^{B}(\tilde{\boldsymbol{k}} \mid \tilde{\boldsymbol{l}})\right|$ will be zero. On the contrary, when $\tilde{\boldsymbol{k}}=\tilde{\boldsymbol{l}}, \operatorname{det}\left|S^{B}(\tilde{\boldsymbol{k}} \mid \tilde{\boldsymbol{l}})\right|$ will be equal to the product of $N-n$ elements of the form $\left(1-s_{i}\right)$, with $i=\tilde{k}_{1}, \ldots, \tilde{k}_{N-n}$, i.e. $\operatorname{det}\left|S^{B}(\tilde{\boldsymbol{k}} \mid \tilde{\boldsymbol{l}})\right|=\Pi_{i \in \tilde{\boldsymbol{k}}}\left(1-s_{i}\right)$. The same applies to $\boldsymbol{S}_{\boldsymbol{k} \boldsymbol{l}}^{A}$, which is given by $\boldsymbol{S}_{\boldsymbol{k} \boldsymbol{l}}^{A}=\operatorname{det}\left|S^{A}(\boldsymbol{k} \mid \boldsymbol{l})\right|=\prod_{i \in \boldsymbol{k}} s_{i}$. This means that if we use Eq. 11 or 12 , the $\boldsymbol{P}$ matrix will be diagonal and equal to $\boldsymbol{S}_{\boldsymbol{k} \boldsymbol{k}}^{A} \tilde{\boldsymbol{S}}_{\boldsymbol{k} \boldsymbol{k}}^{B}$. For a single-determinant wave function, $\hat{\rho}_{n}^{A}$ is then directly in diagonal form, with $\boldsymbol{P}_{\boldsymbol{k} \boldsymbol{k}}=\boldsymbol{P}_{\boldsymbol{k}}=\operatorname{det}\left|S^{A}(\boldsymbol{k} \mid \boldsymbol{k})\right| \times \operatorname{det}\left|S^{B}(\tilde{\boldsymbol{k}} \mid \tilde{\boldsymbol{k}})\right|$, and equal 
to a product of $s_{i}$ and $\left(1-s_{i}\right)$ factors as explained. In this way, for each $\boldsymbol{k}, p_{n}^{\boldsymbol{k}}=\boldsymbol{P}_{\boldsymbol{k}}$ is the product of $N$ factors,

$$
p_{n}^{k}=\prod_{i}^{N} p_{i}
$$

where $p_{i}=s_{i}$ if $i \in \boldsymbol{k}$ and $p_{i}=1-s_{i}$ if $i \in \tilde{\boldsymbol{k}} . \boldsymbol{p}_{n}^{\boldsymbol{k}}$ provides the contribution of $\left|\psi_{\boldsymbol{k}}\right\rangle$ to $p^{A}(n)$, which is finally obtained by adding all the possible arrangements of the $n$ electrons in the the $\left|\psi_{\boldsymbol{k}}\right\rangle$ basis: $\sum_{\boldsymbol{k}} \boldsymbol{p}_{n}^{\boldsymbol{k}}=p^{A}(n)$. With this,

$$
\hat{\rho}_{n}^{A}=\mathbf{1}_{n}^{\prime A} \mathbf{1}_{n}^{A} \times \sum_{\boldsymbol{k}}\left|\psi_{\boldsymbol{k}}\right\rangle \boldsymbol{p}_{n}^{\boldsymbol{k}}\left\langle\psi_{\boldsymbol{k}}\right|,
$$

which is the well-known Schmidth decomposition of a Slater determinant. ${ }^{32}$

Moreover, since in the DNO basis $\boldsymbol{S}_{\boldsymbol{k} \boldsymbol{l}}^{A}$ is diagonal and $\operatorname{det}\left|S^{A}(\boldsymbol{k} \mid \boldsymbol{k})\right|=\prod_{i \in \boldsymbol{k}} s_{i}$, each $n$-electron determinant normalized in $A,\left|\psi_{\boldsymbol{k}}\right\rangle$, can be directly obtained from the normalized spinorbitals $\phi_{i}=\tilde{u}_{i}^{P}$. In terms of these $\phi_{i}$ 's, $\left|\psi_{\boldsymbol{k}}\right\rangle$ is simply a standard determinant $\left|\psi_{\boldsymbol{k}}\right\rangle=$ $(n !)^{-1 / 2}\left|\phi_{k_{1}}\left(\boldsymbol{x}_{1}\right) \phi_{k_{2}}\left(\boldsymbol{x}_{2}\right) \ldots \phi_{k_{n}}\left(\boldsymbol{x}_{n}\right)\right|$.

Normalized DNOs are directly the entanglement orbitals of the system, contributing to the entanglement entropy whenever their eigenvalues $s_{i} \leq 1$, i.e when the $\phi_{i}$ orbital is delocalized among the subsystems. Entanglement in the theory of open quantum systems is thus a measure of chemical bonding, as standardly defined in QCT. In the entanglement orbital or DNO basis all the sector density operators are already in diagonal form.

\subsection{Sector natural orbitals}

The sector RDMs are also easily obtained in the DNO basis. Since Eq. 15 is a sum of single determinant contributions, the standard Löwdin expressions for the RDMs apply to each term. For instance, the sector $1 \mathrm{RDM} \rho_{n}^{A, 1}\left(\boldsymbol{x} ; \boldsymbol{x}^{\prime}\right)$, that can be obtained from Eqs. 5 (if $m=1), 14$, and 15 , is directly diagonal, 


$$
\rho_{n}^{A, 1}\left(\boldsymbol{x} ; \boldsymbol{x}^{\prime}\right)=\mathbf{1}_{1}^{\prime A} \mathbf{1}_{1}^{A} \times \sum_{j=1}^{N} \phi_{j}^{\star}\left(\boldsymbol{x}^{\prime}\right) n_{n, j}^{A, 1} \phi_{j}(\boldsymbol{x}),
$$

where $n_{n, j}^{A, 1}$ are the (not normalized) sector domain natural occupations, $n_{n, j}^{A, 1}=s_{j} \times p_{j}^{A}(n-1)$, with $p_{j}^{A}(n-1)$ representing the probability that $n-1$ electrons lie in $A$ and $N-1-n$ electrons in $B$ for a hypotetical $(N-1)$-electron single determinant wave function built with all $\phi_{i}$ spin-orbitals except $\phi_{j}$. For instance, when $N=4$ and $n=3, p_{1}^{A}(2)=s_{2} s_{3}\left(1-s_{4}\right)+s_{2} s_{4}(1-$ $\left.s_{3}\right)+s_{3} s_{4}\left(1-s_{2}\right), p_{2}^{A}(2)=s_{1} s_{3}\left(1-s_{4}\right)+s_{1} s_{4}\left(1-s_{3}\right)+s_{3} s_{4}\left(1-s_{1}\right)$, etc.

The trace of the sector $1 \mathrm{RDM}$ is equal to $n \times p^{A}(n)$, the contribution of sector $n$ to the domain electron population. The occupation number of DNO $\phi_{i}$ can also be shown to be the overall probability that $\phi_{i}$ is found occupied in all the $\left|\psi_{\boldsymbol{l}}\right\rangle$ determinants in the sector multiplied by the probability that a $\phi_{i}$ electron be found in $A$. If $\tilde{\rho}$ 's are used, then normalized occupation numbers adding to $n$ appear which are just $\tilde{n}_{n, j}^{A, 1}=n_{n, j}^{A, 1} / p^{A}(n)$. This shows that DNOs are the sector natural orbitals for any sector (this ceases to be true in the case that electron correlation is taken into account).

Quite naturally, the total domain $1 \mathrm{RDM}, \rho^{A, 1}\left(\boldsymbol{x} ; \boldsymbol{x}^{\prime}\right)$ is again diagonal in the DNO basis, and $n_{j}^{A, 1}$, the global occupation number of $\phi_{j}$ is simply the sum (if not normalized) or the weighted sum (if normalized) of the sector natural occupations. For instance, $n_{j}^{A, 1}=\sum_{n} p^{A}(n) \tilde{n}_{n, j}^{A, 1}$, the average occupation of the DNO in all sectors. This result shows the consistency of the approach. Since a considerable literature on DNOs and chemical bonding has gathered over the years, ${ }^{33,34}$ we now connect this literature with the concept of entanglement. Moreover, we also notice that all the sector RDMs are particular cases of the so-called coarsed-grained density matrices, introduced in chemical bonding theory to deal with real space resonance structures. ${ }^{27}$ 


\subsection{Sector natural geminals}

The DNO basis can also be exploited to construct the sector geminals. Regarding $\rho_{n}^{A, 2}\left(\boldsymbol{x}_{1}, \boldsymbol{x}_{2} ; \boldsymbol{x}_{1}^{\prime} ; \boldsymbol{x}_{2}^{\prime}\right)$, similar manipulations as those performed in the last subsection lead to

$$
\begin{array}{r}
\rho_{n}^{A, 2}\left(\boldsymbol{x}_{1}, \boldsymbol{x}_{2} ; \boldsymbol{x}_{1}^{\prime} ; \boldsymbol{x}_{2}^{\prime}\right)=\frac{1}{2} A_{12} A_{12}^{\prime} \times \\
\sum_{j, k}^{\prime} \phi_{j}\left(\boldsymbol{x}_{1}\right) \phi_{k}\left(\boldsymbol{x}_{2}\right)\left(n_{n, j, k}^{A, 2}\right) \phi_{j}^{\star}\left(\boldsymbol{x}_{1}^{\prime}\right) \phi_{k}^{\star}\left(\boldsymbol{x}_{2}^{\prime}\right),
\end{array}
$$

where $A_{12}=1-\hat{p}_{12}$ is an operator that antisymmetrizes with respect to variables in the unstarred spin-orbitals, $A_{12}^{\prime}$ acts likewise on the starred spin-orbitals, and $n_{n, j, k}^{A, 2}=s_{j} s_{k} \times$ $p_{j k}^{A}(n-2)$. Now, $p_{j k}^{A}(n-2)$ represents the probability of having $n-2$ electrons in $A$ and $N-n$ electrons in $B$ for a hypotetical $(N-2)$-electron single determinant wave function built with all $\phi_{i}$ spin-orbitals except $\phi_{j}$ and $\phi_{k}$. For instance, in the above example with $N=4$ and $n=2$ we have $p_{12}^{A}(1)=s_{3}\left(1-s_{4}\right)+s_{4}\left(1-s_{3}\right), p_{13}^{A}(1)=s_{2}\left(1-s_{4}\right)+s_{4}\left(1-s_{2}\right)$, etc. This shows that the pairs of DNOs are the sector geminals much as the product of standard orbitals are geminals for Hartree-Fock wavefunctions. The geminal occupation numbers are easily interpreted as the probability of finding a given pair of DNOs in the determinants forming the sector. The trace of the (not normalized) $\rho_{n}^{A, 2}$ is $n(n-1) \times p^{A}(n)$, the contribution of the sector to the average number of pairs in domain $A$. This becomes $n(n-1)$ if normalized $\tilde{\rho}_{n}^{A, 2}$ are used. The arguments here contained are easily generalized to show that any sector RDM is constructed from DNOs using standard rules. In this sense, the set of $\phi_{i}^{\prime} s$ and $s_{i}$ 's contain all the information needed to construct sector densities in the single determinant case. 


\section{The multi-determinant case}

Generalization to multi-determinant wave functions (MDWs) is easy. To extend Eq. 8, we first expand the size of the basis of spin-orbitals $u_{i}$ to a size $m>N$. Then,

$$
|\Psi\rangle=\sum_{r=1}^{M} C_{r}(N !)^{-1 / 2}\left|\Psi_{r}\right\rangle
$$

where $\left|\Psi_{r}\right\rangle=\operatorname{det}\left|u_{1}^{r} \ldots u_{N}^{r}\right|$, and $\left(u_{1}^{r} \ldots u_{N}^{r}\right)$ denotes a subset of the $u_{i}$ 's containing $N$ elements. The indices of the $N$ elements of the subset of $u_{i}$ 's that define $\left|\Psi_{r}\right\rangle$ will be denoted as $r_{1}, \ldots, r_{N}$. Then, we can write

$$
\begin{aligned}
\hat{\rho}_{n}^{A}\left(\boldsymbol{x}_{i \leq n} ; \boldsymbol{x}_{i \leq n}^{\prime}\right) & =\mathbf{1}_{n}^{\prime A} \mathbf{1}_{n}^{A} \times \sum_{r, s}^{M} C_{r} C_{s}^{\star}\left[\hat{\rho}_{n}^{A}\right]_{r s}, \text { with } \\
{\left[\hat{\rho}_{n}^{A}\right]_{r s} } & =\sum_{\boldsymbol{k}, \boldsymbol{l}}\left|U_{\boldsymbol{k}}^{r}\right\rangle\left\langle U_{\boldsymbol{l}}^{s}|\operatorname{det}| S_{r s}^{B}(\tilde{\boldsymbol{k}} \mid \tilde{\boldsymbol{l}})\right|, \\
\left|U_{\boldsymbol{k}}^{r}\right\rangle & =(n !)^{-1 / 2}\left|u_{k_{1}}^{r}\left(\boldsymbol{x}_{1}\right) \ldots u_{k_{n}}^{r}\left(\boldsymbol{x}_{n}\right)\right|, \\
\left\langle U_{\boldsymbol{l}}^{s}\right| & =(n !)^{-1 / 2}\left|u_{l_{1}}^{s}\left(\boldsymbol{x}_{1}^{\prime}\right) \ldots u_{l_{n}}^{s}\left(\boldsymbol{x}_{n}^{\prime}\right)\right| .
\end{aligned}
$$

In the above equations, $\boldsymbol{k}$ and $\boldsymbol{l}$ are $n$-elements subsets of $\left(r_{1} \ldots r_{N}\right)$ and $\left(s_{1} \ldots s_{N}\right)$, respec-

tively, $\tilde{\boldsymbol{k}}$ and $\tilde{\boldsymbol{l}}$ their corresponding $(N-n)$-elements complementary subsets, and $S_{r s}^{B}(\tilde{\boldsymbol{k}} \mid \tilde{\boldsymbol{l}})$ the DOM in $B$ between the spin-orbitals not contained in $\left|U_{\boldsymbol{k}}^{r}\right\rangle$ and the spin-orbitals not contained in $\left\langle U_{l}^{r}\right|$.

The set of $m u_{i}$ 's can again be subjected to the unitary rotation $\boldsymbol{V}^{\dagger} \boldsymbol{S}^{A} \boldsymbol{V}=\operatorname{diag}\left(s_{i}\right)=\boldsymbol{s}$, where $S_{i j}^{A}=\left\langle u_{i} \mid u_{j}\right\rangle_{A}(i, j=1, \ldots, m)$. This renders a new set of functions orthonormal in $R^{3}$ and orthogonal in $A$ and $B$. We will assume that all the $\left|\Psi_{r}\right\rangle$ 's have been rewritten in this basis (this is costly for large $M$ 's and changes the $C_{r}$ coefficients as well as the expansion size $M$, but helps in the discussion). The $u_{i}$ 's then actually satisfy $S_{i j}^{A}=\left\langle u_{i} \mid u_{j}\right\rangle_{A}=s_{i} \delta_{i j}$, $S_{i j}^{B}=\left\langle u_{i} \mid u_{j}\right\rangle_{B}=\left(1-s_{i}\right) \delta_{i j}$, and $S_{i j}=\left\langle u_{i} \mid u_{j}\right\rangle_{R^{3}}=\delta_{i j}, i, j=1, \ldots, m$. This leads again 
to $\tilde{\boldsymbol{k}}=\tilde{\boldsymbol{l}}$ for $S_{r s}^{B}(\tilde{\boldsymbol{k}} \mid \tilde{\boldsymbol{l}}) \mid$ to be nonzero, and to $\operatorname{det}\left|S_{r s}^{B}(\tilde{\boldsymbol{k}} \mid \tilde{\boldsymbol{k}})\right|$ being a product of the $\left(1-s_{i}\right)$ terms already considered. However, this does not lead now to $\boldsymbol{k}=\boldsymbol{l}$, since $\left(r_{1}, \ldots, r_{N}\right)$, the set of $u_{i}$ 's in $\left|U_{\boldsymbol{k}}^{r}\right\rangle$, may differ from $\left(s_{1}, \ldots, s_{N}\right)$, the set of $u_{i}$ 's in $\left\langle U_{\boldsymbol{l}}^{s}\right|$. Sector $n$ will include non-diagonal $\boldsymbol{k} \neq \boldsymbol{l}$ terms differing at most in $n$ spin orbitals. This means that the (unitarily transformed) $u_{i}$ 's do no longer lead to diagonal sectors, and that the entanglement orbitals must be obtained by diagonalizing $\hat{\rho}_{n}^{A}$ in the $\left|U_{k}^{r}\right\rangle$ basis.

This can be done as follows. Starting from the original expansion in Eq. 19, built with non-transformed $u_{i}$ 's, a multi-electron basis $\left|V_{j}\right\rangle$ in sector $n$ is built by ordering all the $n$ electron determinants that can be formed from the $m$ spin-orbitals $u_{i}$. Each $\left|U_{\boldsymbol{k}}^{r}\right\rangle$ determinant in Eq. 19 corresponds to one element of this basis, $\left|U_{\boldsymbol{k}}^{r}\right\rangle \equiv\left|V_{j(r, \boldsymbol{k})}\right\rangle$, this defining the map $j(r, \boldsymbol{k})$. We can now write the matrix of $\hat{\rho}_{n}^{A}$ in the $\left|V_{j}\right\rangle$ basis as

$$
\hat{\rho}_{n}^{A}=\mathbf{1}_{n}^{\prime A} \mathbf{1}_{n}^{A} \sum_{i, j}\left|V_{i}\right\rangle \boldsymbol{T}_{i j}^{B}\left\langle V_{j}\right|,
$$

where

$$
\boldsymbol{T}_{i(r, \boldsymbol{k}), j(s, \boldsymbol{l})}^{B}=\sum_{r s} C_{r} C_{s}^{\star} \operatorname{det}\left|S_{r s}^{B}(\tilde{\boldsymbol{k}} \mid \tilde{\boldsymbol{l}})\right| .
$$

The number of elements in the $\left|V_{j}\right\rangle$ basis is in $\left(\begin{array}{c}m \\ n\end{array}\right)$. Now the basis $|V\rangle$ can be Löwdin orthogonalized, as in our single determinant example, to construct the $n$-electron basis $\left|\psi_{j}\right\rangle=$ $\left|V_{j}\right\rangle\left(\boldsymbol{S}^{A}\right)^{-1 / 2}$, where $\boldsymbol{S}^{A}$ is the domain overlap matrix of the original $|V\rangle$ basis, and the spectrum of the sector will be obtained by diagonalizing $\left(\boldsymbol{S}^{A}\right)^{1 / 2} \boldsymbol{T}^{B}\left(\boldsymbol{S}^{A}\right)^{1 / 2}$ which leads to to the eigenbasis $\left|\phi_{i}\right\rangle$.

The elements of this new basis are linear combinations of the original $\left|\psi_{i}\right\rangle$ ones, and the sector density operator is written as $\hat{\rho}_{n}^{A}=\mathbf{1}_{n}^{\prime} \mathbf{1}_{n}^{A} \sum_{j}\left|\phi_{j}\right\rangle p_{n}^{j}\left\langle\phi_{j}\right|$. The entanglement spectrum of the sector is simply the set of $p_{n}^{j}$ 's. Any of the eigenvalues $p_{n}^{j}$ is interpreted as the probability of finding the $n$ electrons in sector $n$ in the multielectron multi-determinant state $\left|\phi_{j}\right\rangle$. Of course, $\sum_{j} p_{n}^{j}=p^{A}(n)$. The several entanglement entropy measures are immediately obtained from the $p_{n}^{j}$ 's. 


\subsection{Multi-determinant sector natural orbitals and geminals}

A number of simplifications appear if the sector RDMs are built in the DNO-like basis (notice that the CI coefficients $C_{r}$ in Eq. 18 will be different if the $\left|\Psi_{r}\right\rangle$ 's are built in this basis, See the Supplementary Information). Now only $r, s$ determinants differing in one (1RDM) or two (2RDM) spinorbitals at most have to be considered.

In the $1 \mathrm{RDM}$ case, let us write $\rho_{n}^{A, 1}\left(\boldsymbol{x} ; \boldsymbol{x}^{\prime}\right)=\sum_{r, s} C_{r}^{\star} C_{s} \rho_{n, r s}^{A, 1}\left(\boldsymbol{x} ; \boldsymbol{x}^{\prime}\right)$. Simple generalizations lead to

$$
\rho_{n, r r}^{A, 1}\left(\boldsymbol{x} ; \boldsymbol{x}^{\prime}\right)=\sum_{j=1}^{N} u_{r_{j}}^{\star}\left(\boldsymbol{x}^{\prime}\right) u_{r_{j}}(\boldsymbol{x}) p_{r_{j}}^{A}(n-1),
$$

where $r_{1}$ to $r_{N}$ are the orbitals from which determinant $\left|\Psi_{r}\right\rangle$ is built. Similarly, assuming that $\left|\Psi_{r}\right\rangle$ and $\left|\Psi_{s}\right\rangle$ differ in a single spin-orbital, $u_{r_{j}} \neq u_{s_{j}}, \rho_{n, r s}^{A, 1}\left(\boldsymbol{x} ; \boldsymbol{x}^{\prime}\right)$ becomes

$$
\rho_{n, r s}^{A, 1}\left(\boldsymbol{x} ; \boldsymbol{x}^{\prime}\right)=u_{r_{j}}^{\star}\left(\boldsymbol{x}^{\prime}\right) u_{s_{j}}(\boldsymbol{x}) p_{r_{j}}^{A}(n-1) .
$$

In Eqs. 25 and 26, $p_{r_{j}}^{A}(n-1)$ represents the probability of having $n-1$ electrons in $A$ and $N-1-n$ electrons in $B$ for a hypotetical $(N-1)$-electron single determinant wave function built from $\left|\Psi_{r}\right\rangle$ with all $u_{r_{i}}$ 's except $u_{r_{j}}$. Obviously, $p_{r_{j}}^{A}(n-1)=p_{s_{j}}^{A}(n-1)$ if the

single determinant is built by deleting the $u_{s_{j}}$ spin-orbital from $\left|\Psi_{s}\right\rangle$. From this the $\rho^{A, 1}$ matrix is built and diagonalized in the $u_{i}$ one-electron basis. The sector natural orbitals turn out to be linear combinations of the DNO-like functions. Each sector natural orbitals are different.

In the 2RDM case we can equally write $\rho_{n}^{A, 2}=\sum_{r, s} C_{r}^{\star} C_{s} \rho_{n, r s}^{A, 2}$ and find simple expressions for $\rho_{r s}^{A, 2}$ in the three possible $r, s$ cases:

$$
\rho_{n, r r}^{A, 2}=\frac{1}{2} A_{12} A_{12}^{\prime} \sum_{j, k}^{\prime} u_{r_{j}}\left(\boldsymbol{x}_{1}\right) u_{r_{k}}\left(\boldsymbol{x}_{2}\right) u_{r_{j}}^{\star}\left(\boldsymbol{x}_{1}^{\prime}\right) u_{r_{k}}^{\star}\left(\boldsymbol{x}_{2}^{\prime}\right) p_{r_{j} r_{k}}^{A}(n-2)
$$

where $p_{r_{j} r_{k}}^{A}(n-2)$ is the probability of finding $n-2$ electrons in $A$ and $N-n$ electrons in $B$ for a SDW formed from $\Psi_{r}$ (or $\Psi_{s}$ ) by deleting spin-orbitals $u_{r_{j}}$ and $u_{r_{k}}$. 
When $\left|\Psi_{i}\right\rangle$ and $\left|\Psi_{s}\right\rangle$ differ in a single spin-orbital $u_{r_{j}} \neq u_{s_{j}}$, being all the other equal is given by

$$
\rho_{n, r s}^{A, 2}=A_{12} A_{12}^{\prime} \sum_{k \neq j} u_{r_{j}}\left(\boldsymbol{x}_{1}\right) u_{s_{k}}\left(\boldsymbol{x}_{2}\right) u_{s_{j}}^{\star}\left(\boldsymbol{x}_{1}^{\prime}\right) u_{s_{k}}^{\star}\left(\boldsymbol{x}_{2}^{\prime}\right) p_{r_{j} r_{k}}^{A}(n-2)
$$

where $p_{r_{j} r_{k}}^{A}(n-2)$ is the probability of having $n-2$ electrons in $A$ and $N-n$ electrons in $B$ for a SDW formed from $\left|\Psi_{r}\right\rangle$ by deleting the spin-orbital $u_{r_{j}}$ or from $\left|\Psi_{s}\right\rangle$ by deleting the spin-orbital $u_{s_{j}}$.

Finally, when $\left|\Psi_{r}\right\rangle$ and $\left|\Psi_{s}\right\rangle$ differ in two spin-orbitals $u_{r_{j}} \neq u_{s_{j}}$ and $u_{r_{k}} \neq u_{s_{k}}$ we have

$$
\rho_{n, r s}^{A, 2}=A_{12} A_{12}^{\prime} u_{r_{j}}\left(\boldsymbol{x}_{1}\right) u_{r_{k}}\left(\boldsymbol{x}_{2}\right) u_{s_{j}}^{\star}\left(\boldsymbol{x}_{1}^{\prime}\right) u_{s_{k}}^{\star}\left(\boldsymbol{x}_{2}^{\prime}\right) p_{r_{j} r_{k}}^{A}(n-2) .
$$

As before, the $\rho_{n}^{A, 2}$ matrix can now be written in the two-electron basis and diagonalized. Geminals are again sector dependent.

\section{Chemical bonding issues}

As commented in the Introduction, QCT has shown how to reformulate many of the concepts in the theory of the chemical bond in terms of orbital invariant quantities, constructed from spatial density matrices. This has provided a rich arena in which new objects are proposed and used: domain averaged Fermi holes (DAFH), ${ }^{17,18,28,29}$ natural adaptive orbitals (NAdOs), ${ }^{30}$ maximum probability domains, ${ }^{25}$ etc. Over the years, the bonding concepts of chemistry have found their way in QCT. In agreement with conventional wisdom, bonding between two spatial regions is related to electron delocalization: standard covalency appears when delocalization is basically symmetric. Polarity, leading in the limit to charge transfer and ionic behavior, when the delocalization is asymmetric. ${ }^{19,20}$

It has been clear since the inception of QCT that the spatial regions considered in these methods do not contain a fixed number of electrons. ${ }^{4}$ This led to the consideration of domain expectation values (atomic expectation values in the QTAIM). For instance, the atomic net 
charge is defined in the QTAIM as $q_{A}=Z_{A}-\int_{A} \rho^{1}(\boldsymbol{r}) d \boldsymbol{r}$, where $Z_{A}$ is the nuclear charge and $\rho^{1}$ is the electron density. Similarly, a measure of the covalent bond order that quantifies the number of shared pairs of electrons existing between two domains $A$ and $B$ is provided by the delocalization index $\operatorname{DI}(A, B)=\int_{A} d \boldsymbol{r}_{1} \int_{B} d \boldsymbol{r}_{2} \rho_{x c}\left(\boldsymbol{r}_{1}, \boldsymbol{r}_{2}\right)$, with $\rho_{x c}$ being the exchangecorrelation density. ${ }^{35,36}$

Thinner objects have also been defined, as when R. Ponec noticed that the average atomic population $n_{A}$ could be decomposed into one-electron components by diagonalizing the domain averaged charge-weighted Fermi hole. ${ }^{28,29}$

We have effectively shown here that Ponec's DNOs are the eigenvectors of the total domain $1 \mathrm{RDM}, \rho^{A, 1}$, for single determinant functions, so that the DNO label was perfectly chosen. Since in this SDW case the DNOs diagonalize all the sector 1RDMs, Ponec's domain natural occupations are nothing but the weighted sum of the sector eigenvalues, measuring the probability of occupation of each of the entanglement orbitals in the chosen domain. A mostly relevant issue in this SDW case comes from examining the $A, B$ DI. It has also been shown ${ }^{37}$ that $\mathrm{DI}(A, B)=2 \sum_{i} n_{i}^{A, 1}\left(1-n_{i}^{A, 1}\right)$, so that each DNO provides an additive contribution to the covalent bond order that is defined in terms of its degree of delocalization between the $A, B$ region. A purely $A$ - or $B$-localized DNO, with $n^{A, 1}=1,0$, respectively, does not contribute to the DI. The contribution of a given DNO to the DI peaks at $1 / 2$ when it is fully delocalized, $n^{A, 1}=1 / 2$, so that if doubly occupied yields a contribution of 1 to the bond order. In agreement with Tubman and Yang, bonding comes from delocalization in chemical terms, or from entanglement of the spatial regions, in the open systems parlance. The well-known fact that the DNOs of subsystem $A$ are complementary to those of subsystem $B$ with the same occupation number is a simple consequence of the fact that the entanglement spectrum of both subsystems must be equal. The $n^{A, 1}$ values can be used to reconstruct the weight of each sector, the total atomic population, and the bonding strength of subsystem $A$ with $B$.

Electron correlation destroys the simple identification between the eigenvectors of the 
domain averaged Fermi hole and the domain natural orbitals. Actually, the meaning of Ponec's DNOs (which have been generalized and called first order natural adaptive orbitals, 1-NAdOs) ${ }^{30}$ has been examined. ${ }^{17}$ The 1-NAdOs occupations keep on adding to the average domain electron population, $n^{A}$, but need not be positive definite. Negative occupation orbitals provide a window into the role of correlation, but lack an immediate physical correlate. The treatment developed here shows how to obtain sector domain natural orbitals as well as overall DNOs in the fully correlated case. Their occupations also add to $n^{A}$, but are now positive definite.

Finally, we abound on the relation between the sector weights and the electron distribution functions. $p^{A}(n)$ provides the probability of finding $n$ electrons in domain $A$ (and that of finding $N-n$ electrons in $B$ ). It has been found that the distributions are much simpler than expected in the case of atomic partitions, being close to binomial in many standard cases. This implies that only a few electrons are effectively entangled, and that the rest do not participate in the Hilbert space expansion that accompanies the interaction of regions $A$ and $B$. The entangled and unentangled sets of electrons are the standard delocalized/localized or bonding/core sets used in chemistry. A note of caution is due. Since no Schmidt decomposition exists for more than two subsystems, ${ }^{38}$ multi-domain EDFs, which provide insights into multi-center bonding, ${ }^{39}$ are not easily embedded in the present formalism.

The use of sector expectation values for general operators, as briefly sketched with the electron density case, is also possible. This is intimately related with work on coarse-grained density matrices, ${ }^{27}$ but outside the scope of this introductory work. Work along this line is currently being developed.

\section{Computational implementation and toy examples}

The naïve computational implementation of these ideas is formally simple, since every object defined depends ultimately on the domain overlap matrix $S_{i j}^{A}=\left\langle u_{i} \mid u_{j}\right\rangle_{A}$ of all the 
spin-orbitals found in the determinant(s) used to build the wave function. Due to the combinatorial nature of the number of elements in the $\left|U_{\boldsymbol{k}}\right\rangle$ sector bases, and the possibly large number of CI terms in a good quality correlated $|\Psi\rangle$, the scaling of this simple implementation makes computationally very demanding its application to anything but very simple cases. A similar situation is faced when building EDFs for MDWs, although clever algorithms ${ }^{16,25}$ improve the computational complexity. A way out, as TY showed is to rely on quantum Monte Carlo calculations.

Fortunately, and regarding the chemical bonding purposes pursued in QCT, it will be many times sufficient to deal with sector averaged quantities, and not with the full sector machinery. Given the importance of natural expansions, we exemplify this with the sector averaged first order density matrix of a subsystem, together with its eigenvalues and eigenvectors.

As shown, the sector averaged $1 \mathrm{RDM}, \rho^{A, 1}\left(\boldsymbol{x} ; \boldsymbol{x}^{\prime}\right)=\sum_{n} p^{A}(n) \rho_{n}^{A, 1}\left(\boldsymbol{x} ; \boldsymbol{x}^{\prime}\right)$ can be simply written as $\rho^{A, 1}\left(\boldsymbol{x} ; \boldsymbol{x}^{\prime}\right)=\mathbf{1}_{1}^{\prime A} \mathbf{1}_{1}^{A} \rho^{1}\left(\boldsymbol{x} ; \boldsymbol{x}^{\prime}\right)$. To obtain its natural orbitals we simply write its matrix in a given basis and diagonalize it. Let $\left|u_{i}\right\rangle$ be the canonical orbital basis and $\hat{\rho}^{1}=\sum_{i j}\left|u_{i}\right\rangle \rho_{i j}\left\langle u_{j}\right|$. Then the matrix of $\rho^{A, 1}$ in this basis is simply $\rho^{A}=S^{A} \rho S^{A}$. Since the basis is not orthonormal in $A$, we need to solve a non-orthogonal eigenvalue problem, which is equivalent to diagonalizing the $\left(S^{A}\right)^{1 / 2} \rho\left(S^{A}\right)^{1 / 2}$ matrix. Notice that in the SDW case $\rho$ is the unit matrix, and the natural orbitals of the $A$ subsystem are obtained after diagonalizing $S^{A}$, as known. Since both $S^{A}$ and $\rho$ are definite positive, the natural occupations $n_{j}^{A, 1}$ satisfy $0 \leq n_{j}^{A, 1} \leq 1$. In this sense, these DNOs admit a simpler chemical interpretation than those defined by Ponec in the case of correlated wave functions, which can lead to negative occupation numbers. We will refer to the present DNO, and not to Ponec's in the rest of this section.

We stress now that as dynamical electron correlation becomes more and more important in the description of a chemical system, the identification of entanglement and bonding dilutes. In a SDW, $n_{j}^{A, 1}$ identifies bonding. An entangled orbital means a delocalization 
channel. On the contrary, for a correlated MDW, a multitude of lowly populated DNO simply indicates correlation contributions. A critical exam of a similar situation has already been done. ${ }^{40}$

A few toy systems may serve to exemplify our results. We start with $\mathrm{H}_{2}$. Any real space partition is equivalent, since the quantum atoms in a homodiatomic are determined by symmetry. At the spinless HF level the only $\sigma_{g}$ orbital is directly the DNO, with $n^{A, 1}=1$ (or 0.5 if the spin-orbital is considered). This implies a single entangled contribution or delocalization channel, coresponding to the traditional chemical bonding picture of dihydrogen. The weights of the three sectors ( $n=0,1,2$ electrons) are binomial, and equal to $1 / 4,1 / 2,1 / 4$, respectively. ${ }^{16}$

A full configuration interaction (FCI) calculation performed with the GAMESS code ${ }^{41}$ using a TZV $++(2 \mathrm{p}, 2 \mathrm{~d}, \mathrm{f})$ basis yields a (global) natural orbital decomposition with 5 dominating natural orbitals. Their occupations $n^{1}$ are equal to $1.965,0.020,6.1 \times 10^{-3}, 3.9 \times$ $10^{-3}, 3.9 \times 10^{-3}$, that correspond to $\sigma_{g, 1 s}, \sigma_{u, 1 s}, \sigma_{g, 2 s}, \pi_{g, 2 p}, \pi_{u, 2 p}$ components, respectively. If we now diagonalize the $1 \mathrm{RDM}$ in the $A$ region (both $\mathrm{H}$ atoms are equivalent), five components appear again, with $n^{A, 1}=0.990,3.3 \times 10^{-3}, 2.3 \times 10^{-3}, 2.0 \times 10^{-3}, 2.0 \times 10^{-3}$. These are now more or less localized functions that may be identified with $\sigma_{g, 1 s}, \sigma_{g, 2 s}, \sigma_{g, 2 p}, \pi_{g, 2 p}, \pi_{u, 2 p}$ contributions, respectively.

As we can see, a dominant entanglement contribution appears, followed by a set of much smaller contributions, which we identify with correlating terms. The $\sigma_{g, 1 s}$ and $\pi$ DNOs are only slightly more localized in region $A$ than their global counterparts (notice, in any case, that strictly speaking, the DNOs exist only in $A$ ). The other two are much more localized hybrids. It is interesting that we can interpret the $\sigma_{u, 1 s}$ global natural orbital as having been absorbed in the localization procedure to give rise to a slightly localized $\sigma_{g, 1 s}$ DNO in domain $A$ and its equivalent counterpart in domain $B$. In this way the occupation number of the leading DNO (0.990) is larger than half that of the leading global natural orbital $(1.965 / 2=$ 0.983). This is what we expect from chemical intuition, since correlation decreases slightly 
the covalency of the $\mathrm{H}_{2}$ link, which is equivalent to forcing domain localization. This is in agreement with the modification of the sector weights, that change to $0.213,0.574,0.213$ for $n=0,1,2$ electrons, respectively, showing that the probability of finding localized electrons has increased (slightly).

Actually, if we stretch the molecule, the $\sigma_{g, 1 s}$ global occupation decreases as the $\sigma_{u, 1 s}$ one increases. At the dissociation limit, only these two global natural orbitals remain, with degenerate occupations equal to 1 . In the DNO description their mixing leads to a $1 s$ orbital centered at $A$ (or $B$ ), with occupation again equal to one. In the spinless description at dissociation the two electrons have become uncoupled. This is no longer true if spin-DNOs are used, since spin entanglement remains.

A less naïve case is provided by the tetrahedral $\left[\mathrm{PtO}_{4}\right]^{2+}$ cation, which recently raised attention due to its alleged X oxidation state. ${ }^{42}$ We have performed Heat Batch CI (HCI) calculations with the PySCF suite ${ }^{43}$ and the def2-qzvpd (O) and adzp (Pt) basis sets, ${ }^{44}$ and analyzed the results using QTAIM atoms. The QTAIM net charge of the Pt atom is +2.838 $e$, with slightly negatively charged oxygens. An EDF analysis shows that the most probable electron distribution is that with 76 electron in the Pt domain and 8 electrons in each of the $\mathrm{O}$ basins $(p=0.0217)$, closely followed by those with an extra electron in one of the O's $(p=0.0211)$. The probability of all the $\mathrm{O}$ atoms displaying 10 electrons is negligible (smaller than $10^{-5}$ ), and that of finding just one $\mathrm{O}^{2-}$ around $p=0.006$. This immediately shows that a $\mathrm{X}$ formal oxidation number is far from reflecting the electron structure of the system. A look at the correlated DNOs provides useful information. Occupation numbers are shown in Table 1, and isosurfaces of some representants in Fig. 1.

All $1 \mathrm{~s}$ to $5 \mathrm{~s}, 2 \mathrm{p}$ to $4 \mathrm{p}$, and $3 \mathrm{~d}$ to $4 \mathrm{~d}$ occupations are essentially equal to 2 . The $\mathrm{Pt}$ 4f electrons are also completely localized, not engaged in bonding (or entanglement). The 5 p occupations are very close to 2 , and a look at their shape shows that the DNOs have no appreciable ligand contribution. The deviation of their occupations with respect to 2 is thus a correlation, not bonding effect. The first set of orbitals enganged in strong bonding 


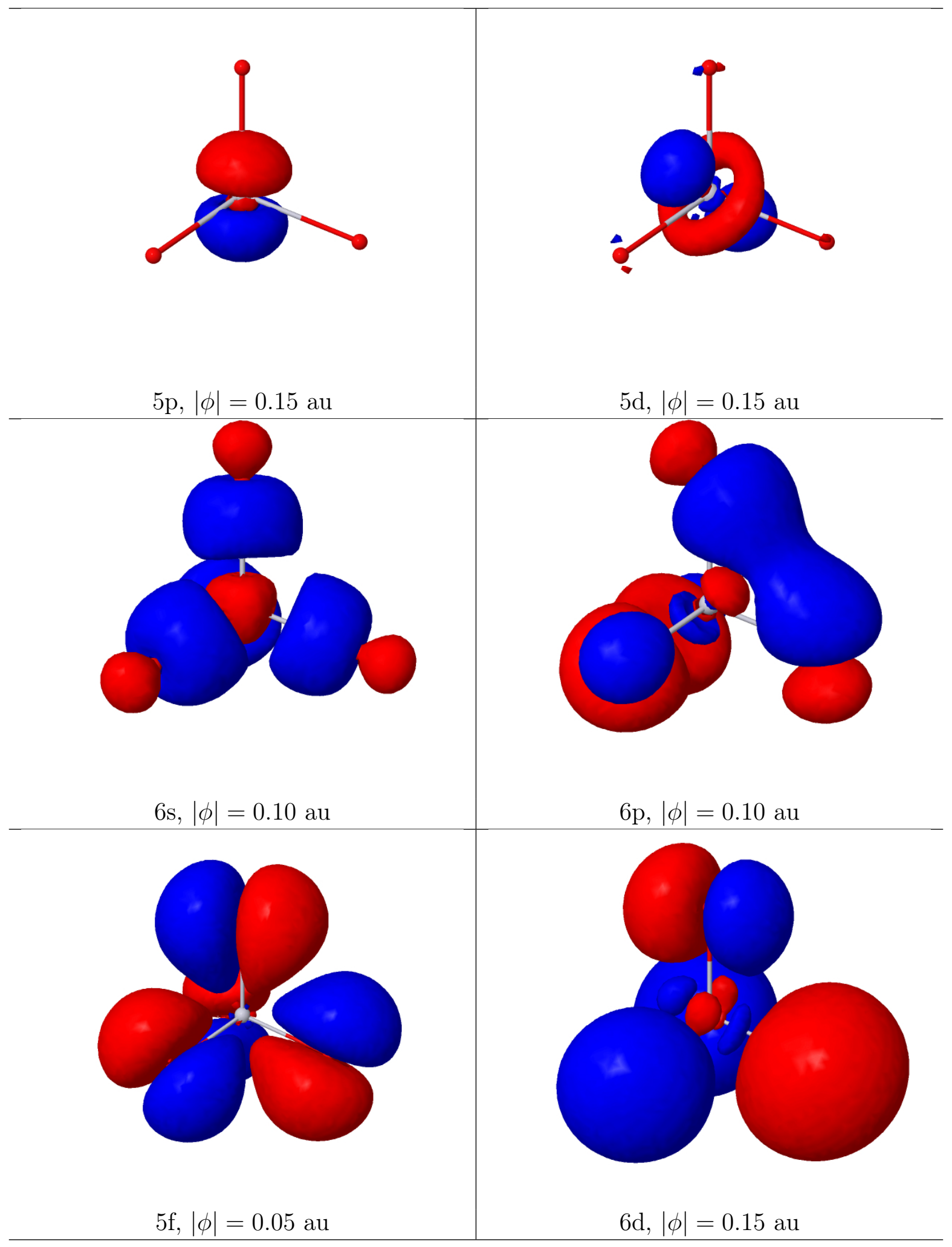

Figure 1: Isosurfaces of relevant Pt DNOs (normalized in $R^{3}$, shown in $R^{3}$ ) in the $\left[\mathrm{PtO}_{4}\right]^{2+}$ complex. 
Table 1: Spinless domain occupation numbers, $n^{A, 1}$ for the $\mathrm{Pt}$ domain in the $\left[\mathrm{PtO}_{4}\right]^{2+}$ system, computed as detailed in the text. Only DNOs with occupations differing from either 2 or 0 in more than 0.001e are shown. Most of the DNOs have a clear atomic character. Labels with an asterisk distinguish the DNOs with large ligand contributions.

\begin{tabular}{cccc}
\hline $5 \mathrm{p}$ & $5 \mathrm{~d}-t_{2}$ & $5 \mathrm{~d}-e$ & $6 \mathrm{~s}^{*}$ \\
\hline 1.986 & 1.139 & 1.343 & 0.353 \\
\hline $6 \mathrm{p}^{*}$ & $5 \mathrm{f}-t_{2}{ }^{*}$ & $6 \mathrm{~d}-e^{*}$ & $6 \mathrm{~d}-t 2^{*}$ \\
\hline 0.188 & 0.050 & 0.002 & 0.011 \\
\hline
\end{tabular}

is the $5 \mathrm{~d}$ manifold. As seen from the Table, their overall population $(6.103 \mathrm{e})$ points to a $\mathrm{d}^{6}$-like configuration. As expected from tetrahedral symmetry, the 5d-e components are more localized in the Pt atom. The 6s-like orbital is heavily mixed with and delocalized over the ligands. All the $6 \mathrm{p}, 5 \mathrm{f}-t 2$ and $6 \mathrm{~d}$ orbitals have also small but sizeable occupations. The participation of $6 \mathrm{p}$-like and 5 f-like functions is to be noticed. Overall they account for about $0.56+0.15=0.71$ of the Pt electrons. This uncovers the multiconfigurational character of the complex, and the importance of $6 \mathrm{p}, 5 \mathrm{f}$ excitations in bonding. On the contrary, we should consider the $6 \mathrm{~d}$ participation as due to mainly dynamical correlation.

\section{Conclusions and prospects}

Quantum chemical topology (QCT) offers a theory of chemical bonding through orbital invariant descriptors. This is achieved by partitioning the real space into regions identified with atoms, bonds, lone pairs, etc. As an example, in the quantum theory of atoms in molecules (QTAIM) the gradient field of the electron density is used to exhaustively partition the space into atomic domains, called quantum atoms. These regions were soon recognized as open quantum systems, ${ }^{4}$ with a fluctuating number of particles, for instance. However, QCT has not been studied through the lens of the general theory of open quantum systems. We have shown in this paper how this can be done.

Closed expressions for a subsystem's density operator in real space have been derived by tracing out the degrees of freedom in its complementary region, and the Schmidt de- 
composition (examined in chemical bonding by Tubman and Yang ${ }^{14}$ for instance) has been shown to lead in the single determinant case to Ponec's domain natural orbitals. Moreover, the subsystem density operator can be written as a direct sum of fixed number of electrons sectors, with weights that turn out to be exactly equal to those of the electron distribution functions (EDFs). ${ }^{16}$ Since chemical bonding can be understood in terms of the statistical cumulant moments of EDFs, ${ }^{39}$ a deep link is established between chemical concepts, like bonding channels, and the mutual entanglement between the subsystems.

We have also shown that all expectation values can be written as weighted averages of sector expectations, in agreement with previous analyses that used coarse-grained density

matrices. ${ }^{27}$ As an example, we have examined the global first order density matrix of a subsystem and its eigensolution in a couple of toy systems. In the multi-determinant wave function case, the domain natural orbitals defined through this open systems approach do not coincide with those of Ponec's.

Although no Schmidt decomposition for more than two complementary systems exists in general, a multi-domain real space description is important in chemistry, where multi-center bonding concepts appear. We think that work along that direction should be done in the near future.

\section{Acknowledgement}

We thank the spanish MINECO/FEDER, grant CTQ2015-65790-P.

\section{Supporting Information Available}

Expansion of a multideterminant wavefunction in terms of two orbital bases related by an unitary transformation 


\section{References}

(1) Gimarc, B. M. Molecular structure and bonding. The qualitative molecular orbital approach; Academic Press: New York, 1979.

(2) Popelier, P. L. A. In Structure and Bonding, Vol 115.; Wales, D. J., Ed.; Springer, Berlin, 2005; Chapter Quantum Chemical Topology: On Bonds and Potentials, pp $1-56$.

(3) Levy, D. E. Arrow-pushing in organic chemistry : an easy approach to understanding reaction mechanisms; John Wiley \& Sons, Inc: Hoboken, NJ, 2017.

(4) Bader, R. F. W. Atoms in Molecules; Oxford University Press: Oxford, 1990.

(5) Amico, L.; Fazio, R.; Osterloh, A.; Vedral, V. Entanglement in many-body systems. Rev. Mod. Phys. 2008, 80, 517-576.

(6) Li, H.; Haldane, F. D. M. Entanglement Spectrum as a Generalization of Entanglement Entropy: Identification of Topological Order in Non-Abelian Fractional Quantum Hall Effect States. Phy. Rev. Lett. 2008, 101, 010504-1-010504-4.

(7) Haque, M.; Zozulya, O.; Schoutens, K. Entanglement Entropy in Fermionic Laughlin States. Phys. Rev. Lett. 2007, 98, 060401-1-060401-4.

(8) Fang, C.; Gilbert, M. J.; Bernevig, B. A. Entanglement spectrum classification ofCninvariant noninteracting topological insulators in two dimensions. Phys. Rev. B 2013, 87, 035119-1-035119-17.

(9) Knizia, G.; Chan, G. Density Matrix Embedding: A Simple Alternative to Dynamical Mean-Field Theory. Phys. Rev. Lett. 2012, 109, 186404-1,186404-5.

(10) Knizia, G.; Chan, G. Density Matrix Embedding: A Strong-Coupling Quantum Embedding Theory. J. Chem. Theory Comput. 2013, 9, 1428-1432. 
(11) White, S. R.; Martin, R. L. Ab initio quantum chemistry using the density matrix renormalization group. J. Chem. Phys. 1999, 110, 4127-4130.

(12) Boguslawski, K.; Tecmer, P.; Örs Legeza,; Reiher, M. Entanglement Measures for Single- and Multireference Correlation Effects. The Journal of Physical Chemistry Letters 2012, 3, 3129-3135.

(13) Boguslawski, K.; Tecmer, P. Orbital entanglement in quantum chemistry. International Journal of Quantum Chemistry 2014, 115, 1289-1295.

(14) Tubman, N. M.; Yang, D. C. Calculating the entanglement spectrum in quantum Monte Carlo with application toab initioHamiltonians. Physical Review B 2014, 90, 081116$1-081116-5$.

(15) Tubman, N. M.; Yang, D. C. Quantum dissection of a covalent bond with the entanglement spectrum. https://arxiv.org/abs/1412.1495, 2014.

(16) Francisco, E.; Martín Pendás, A.; Blanco, M. A. Electron Number Probability Distributions for Correlated Wave Functions. J. Chem. Phys. 2007, 126, 094102-1-094102-13.

(17) Bochicchio, R.; Ponec, R.; Lain, L.; Torre, A. Pair Population Analysis within AIM Theory. J. Phys. Chem. A 2000, 104, 9130-9135.

(18) Bochicchio, R. C.; Ponec, R.; Torre, A.; Lain, L. Multicenter bonding within the AIM theory. Theor. Chem. Acc. 2001, 105, 292-298.

(19) Blanco, M. A.; Martín Pendás, A.; Francisco, E. Interacting Quantum Atoms: A Correlated Energy Decomposition Scheme Based on the Quantum Theory of Atoms in Molecules. J. Chem. Theory Comput. 2005, 1, 1096-1109.

(20) Francisco, E.; Martín Pendás, A.; Blanco, M. A. A Molecular Energy Decomposition Scheme for Atoms in Molecules. J. Chem. Theory Comput. 2006, 2, 90-102. 
(21) Breuer, H.-P.; Petruccione, F. The theory of open quantum systems; Oxford University Press: Oxford New York, 2002.

(22) Nielsen, M.; Chuang, I. L. Quantum computation and quantum information; Cambridge University Press: Cambridge New York, 2010.

(23) Ekert, A.; Knight, P. L. Entangled quantum systems and the Schmidt decomposition. Am. J. Phys. 1995, 63, 415-423.

(24) Daudel, R. The Fundamentals of Theoretical Chemistry; Pergamon Press: Oxford, 1968.

(25) Cancès, E.; Keriven, R.; Lodier, F.; Savin, A. How electrons guard the space: shape optimization with probability distribution criteria. Theor. Chem. Acc. 2004, 111, 373380.

(26) Martín Pendás, A.; Francisco, E.; Blanco, M. A. An Electron Number Distribution View of Chemical Bonds in Real Space. Phys. Chem. Chem. Phys. 2007, 9, 1087-1092.

(27) Martín Pendás, A.; Francisco, E.; Blanco, M. A. Pauling Resonant Structures in Real Space through Electron Number Probability Distributions. J. Phys. Chem. A 2007, 111, 1084-1090.

(28) Ponec, R. Electron pairing and chemical bonds. Chemical structure, valences and structural similarities from the analysis of the Fermi holes. J. Math. Chem. 1997, 21, 323333.

(29) Ponec, R. Electron pairing and chemical bonds. Molecular structure from the analysis of pair densities and related quantities. J. Math. Chem. 1998, 23, 85-103.

(30) Menéndez, M.; Boto, R. Á.; Francisco, E.; Martín Pendás, A. One-electron images in real space: Natural adaptive orbitals. J. Comput. Chem. 2015, 36, 833-843.

(31) Martín Pendás, A.; Francisco, E.; Blanco, M. A. Spin resolved electron number distribution functions: How spins couple in real space. J. Chem. Phys. 2007, 127, 144103. 
(32) Peschel, I. Special Review: Entanglement in Solvable Many-Particle Models. Braz. J. Phys. 2012, 42, 267-291.

(33) Cooper, D. L.; Ponec, R. A one-electron approximation to domain-averaged Fermi hole analysis. Phys. Chem. Chem. Phys. 2008, 10, 1319-1329.

(34) Ponec, R.; Cooper, D. L. Anatomy of bond formation. Bond length dependence of the extent of electron sharing in chemical bonds from the analysis of domain-averaged Fermi holes. Faraday Discuss. 2007, 135, 31-42.

(35) Fradera, X.; Austen, M. A.; Bader, R. F. W. The Lewis Model and Beyond. J. Phys. Chem. A 1999, 103, 304-314.

(36) Outeiral, C.; Vincent, M. A.; Martín Pendás, A.; Popelier, P. L. A. Revitalizing the concept of bond order through delocalization measures in real space. Chemical Science 2018, 9, 5517-5529.

(37) Francisco, E.; Martín Pendás, A.; Blanco, M. A. A connection between domain-averaged Fermi hole orbitals and electron number distribution functions in real space. J. Chem. Phys. 2009, 131, 124125-1-124125-11.

(38) Peres, A. Higher order Schmidt decompositions. Phys. Lett. A 1995, 202, 16-17.

(39) Francisco, E.; Martín Pendás, A.; García-Revilla, M.; Álvarez Boto, R. A Hierarchy of Chemical Bonding Indices in Real Space from Reduced Density Matrices and Cumulants. Comput. Theor. Chem. 2013, 1003, 71-78.

(40) Francisco, E.; Martín Pendás, A.; Costales, A. On the interpretation of domain averaged Fermi hole analyses of correlated wavefunctions. Phys. Chem. Chem. Phys. 2014, 16, 4586-4597.

(41) Schmidt, M. W.; Baldridge, K. K.; Boatz, J. A.; Elbert, S. T.; Gordon, M. S.; Jensen, J. H.; Koseki, S.; Matsunaga, N.; Nguyen, K. A.; Su, S. J.; Windus, T. L.; 
Dupuis, M.; Montgomery, J. A. General Atomic and Molecular Electronic Structure System. J. Comput. Chem. 1993, 14, 1347-1363.

(42) Yu, H. S.; Truhlar, D. G. Oxidation State 10 Exists. Angew. Chem. Int. Ed. 2016, 55, 9004-9006.

(43) Sun, Q.; Berkelbach, T. C.; Blunt, N. S.; Booth, G. H.; Guo, S.; Li, Z.; Liu, J.; McClain, J.; Sharma, S.; Wouters, S.; Chan, G. K.-L. The Python-based Simulations of Chemistry Framework (PySCF). http://arxiv.org/abs/1701.08223, 2017.

(44) Schuchardt, K. L.; Didier, B. T.; Elsethagen, T.; Sun, L.; Gurumoorthi, V.; Chase, J.; Li, J.; Windus, T. L. Basis Set Exchange: A Community Database for Computational Sciences. Journal of Chemical Information and Modeling 2007, 47, 1045-1052. 


\title{
Quantum Chemical Topology as a Theory of Open Quantum Systems
}

\section{ELECTRONIC SUPPLEMENTARY INFORMATION}

$$
\text { by }
$$

\author{
Ángel Martín Pendás* and Evelio Francisco \\ Departamento de Química Física y Analítica. Facultad de Química. \\ Universidad de Oviedo. 33006-Oviedo. Spain. \\ Email: ampendas@uniovi.es \\ Email: evelio@uniovi.es
}

\section{Contents}

1. Expansion of a multideterminant wavefunction in terms of two orbital bases related by an unitary transformation 


\section{Expansion of a multideterminant wavefunction in terms of two orbital bases related by an unitary transformation}

We proof in this supplementary information how both single- and multi-determinant wave functions, initially expressed in a canonical orbital basis $u_{i}(i=1, m),\left\langle u_{i} \mid u_{j}\right\rangle_{R^{3}}=\delta_{i j}$, can also be built in a new basis $u_{i}^{p}$, related to the $u_{i}$ 's by an unitary transformation, which is ortonormal in $R^{3}$ and orthogonal in two complementary domains $A \cup B=R^{3}$. Let $\mathbf{S}^{A}$ the $(m \times m)$ Hermitian matrix defined by $\mathbf{S}_{i j}^{A}=\left\langle u_{i} \mid u_{j}\right\rangle_{A}$ or, in matrix form, $\mathbf{S}^{A}=\langle\boldsymbol{u} \mid \boldsymbol{u}\rangle_{A}$. Now, we define $\mathbf{u}^{p}=\boldsymbol{u} \mathbf{U}$ where $\mathbf{U}$ is the unitary matrix that diagonalizes $\mathbf{S}^{A}, \mathbf{S}^{A} \mathbf{U}=\mathbf{U} \operatorname{diag} \boldsymbol{s}_{A}$. Then

$$
\left\langle\mathbf{u}^{p} \mid \mathbf{u}^{p}\right\rangle_{A}=\mathbf{U}^{\dagger}\langle\boldsymbol{u} \mid \boldsymbol{u}\rangle_{A} \mathbf{U}=\mathbf{U}^{\dagger} \mathbf{S}^{A} \mathbf{U}=\operatorname{diag}\left(\mathbf{s}_{A}\right) .
$$

Given that $\mathbf{S}^{B}=\langle\boldsymbol{u} \mid \boldsymbol{u}\rangle_{B}=\operatorname{diag}(\mathbf{I})-\mathbf{S}^{A}$, $\mathbf{U}$ also diagonalizes $\mathbf{S}^{B}$ with $\operatorname{diag}\left(\mathbf{s}_{B}\right)=\operatorname{diag}(\mathbf{I}-$ $\mathbf{s}_{A}$ ) being their eigenvalues. The basis $\mathbf{u}^{p}$ is thus the same no matter one diagonalizes $\mathbf{S}^{A}$ or $\mathbf{S}^{B}$, and it is simultaneously orthonormal in $\mathbf{R}^{3}$ and orthogonal in $A$ and $B$. Now, let $\mathbf{U}_{\boldsymbol{r} \boldsymbol{j}}$ a $(N \times N)$ matrix $(N \leq m)$ formed by the rows $r_{1} \ldots r_{N}=\boldsymbol{r}$ and the columns $j_{1} \ldots j_{N}=\boldsymbol{j}$ of $\mathbf{U}$, and $|\Psi\rangle$ a $N$-electron wave function given by

$$
|\Psi\rangle=\sum_{r=1}^{M} C_{r}(N !)^{-1 / 2}\left|\psi_{r}\right\rangle,
$$

where $\left|\psi_{r}\right\rangle=\operatorname{det}\left|u_{r_{1}}(1) u_{r_{2}}(2) \ldots u_{r_{N}}(N)\right|$. Using $\boldsymbol{u}=\mathbf{u}^{p} \mathbf{U}^{\dagger}$ and elementary properties of the determinants, it trivial to show that

$$
\left|\psi_{r}\right\rangle=\sum_{j} \operatorname{det}\left(\mathbf{U}_{r j}\right) \operatorname{det}\left|u_{j_{1}}^{p}(1) \ldots u_{j_{N}}^{p}(N)\right|
$$

In the above equation $\boldsymbol{j}$ runs over the $\left(\begin{array}{c}m \\ N\end{array}\right)$ ordered sub-sets that can be extracted from the basis $\mathbf{u}^{p}$. Using Eq. 3 in Eq. 2 we have

$$
|\Psi\rangle=\sum_{j} D_{j}(N !)^{-1 / 2} \operatorname{det}\left|u_{j_{1}}^{p}(1) \ldots u_{j_{N}}^{p}(N)\right| .
$$

with

$$
D_{\boldsymbol{j}}=\sum_{r=1}^{M} C_{r} \operatorname{det}\left(\mathrm{U}_{\boldsymbol{r} \boldsymbol{j}}\right) .
$$

This completes the proof. An interesting point related to Eq. 3 is the following. In general, $\mathbf{U}_{\boldsymbol{r} \boldsymbol{j}}$ is not unitary, so that $\operatorname{det}\left(\mathbf{U}_{\boldsymbol{r} \boldsymbol{j}}\right) \neq 1$. However, for a single-determinant wave function $m=N, \boldsymbol{j}=\boldsymbol{r}=(1,2, \ldots, N)$, and $\mathbf{U}_{\boldsymbol{r} \boldsymbol{j}}$ coincides with $\mathbf{U}$, which is unitary. Consequently, in this case, $\operatorname{det}\left(\mathrm{U}_{\boldsymbol{r} \boldsymbol{j}}\right)=1$ and $\psi_{r}=\operatorname{det}\left|u_{1}^{p}(1) \ldots u_{N}^{p}(N)\right|$. This shows the well-known invariance of an Slater determinant under an unitary transformation of all of its spin-orbitals. 